\title{
Automated Seamless Mosaicking of Multi-Strip Data from CBERS-02C Imagery
}

\author{
Qi Chen \\ School of Remote Sensing and Information Engineering \\ Wuhan University \\ 129 Luoyu Road, Wuhan 430079, China \\ daoqiqi@gmail.com
}

\author{
Mingwei Sun \\ School of Remote Sensing and Information Engineering \\ Wuhan University \\ 129 Luoyu Road, Wuhan 430079, China \\ mingweis@qq.com
}

\begin{abstract}
Since CBERS-02C was successfully launched on December 22, 2011, the data of this satellite has been widely used in many fields. One of the most applicable products is the highresolution mosaicked image which is covered by a combination of 6 charge-coupled device (CCD) line sensors, each with 4096 pixels. This paper proposes an automated seamless mosaicking method to stitch the 6 strips of images efficiently. The entire process employs technical methods including image geometric correction based on Rational Function Model (RFM), image mosaicking and weight-based image feathering. The three processes can be realized in one step without any second resampling of the data. The experimental result indicates that the method is feasible and of relatively high efficiency. The presented method has also been successfully applied to the automatic data processing system of CBERS-02C satellite.
\end{abstract}

Index Terms - CBERS-02C, multi-strip images, geometrical correlation, seamless mosaicking.

\section{INTRODUCTION}

As the first land resources monitoring satellite of China, CBERS-02C is equipped with a multispectral (MUX) camera with ground geometric resolution of $10 \mathrm{~m}$, a panchromatic (PAN) camera with resolution of $5 \mathrm{~m}$, and a panchromatic high-resolution (HR) camera with $2.36 \mathrm{~m}$ resolution. Basically the same as CBERS-02B, HR camera has three CCDs installed in the same optics, and each CCD has 4096 detector elements [1]. The different is that, CBERS-02C is loaded with two identical HR cameras. By combining the two cameras' coverage, a data product of wider range can be achieved.

The high resolution data imaging from CBERS-02C comprises the data of HR-A camera and HR-B camera (Fig.1). Each camera contains three arrays of CCDs, which results in 3 strips of images with an overlap of 20 pixels between adjacent strips. Besides, there is also an overlap of around 600-700 pixels between HR-A and HR-B camera, due to the accuracy of the orientation parameters. Therefore, not only strips of images inside one camera should be considered, the strips from another camera should also be stitched seamlessly to generate a complete scene of CBERS-02C high-resolution image.

An existing method of mosaicking multi-strip data from CBERS-02B, was to perform image matching in overlapped area of images, and then stitch the images by simple shifting with the matching result [1]. It is obvious that the method will not be feasible in mosaicking strip data of HR-A and HR-B, because the photographing directions of the two cameras are not perfectly parallel, which means the bias between A3 and B1 strip could be varying as going down the strip. A targeted algorithm has been proposed to solve this problem, which detected common features in the overlapping area using attitude information along with image correlation theory, then stitched the images successfully by strip-to-strip registration based on a fifth order polynomial [2]. It has also been suggested that high order polynomials are not preferred in strip-to-strip registration, the recommended order is two [3].

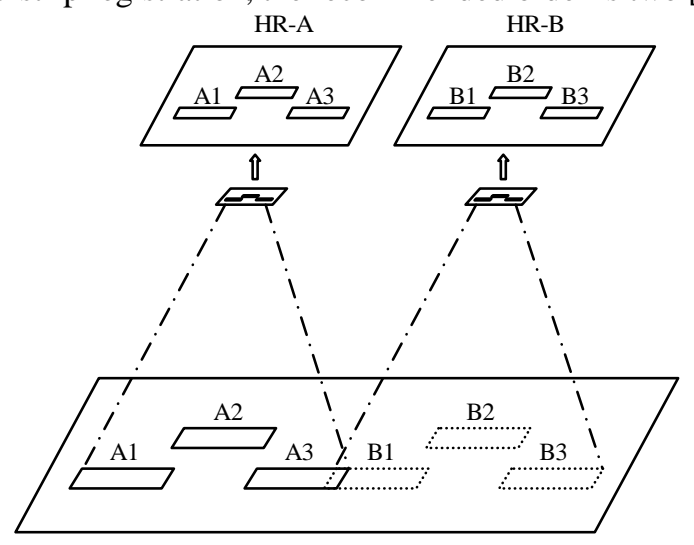

Fig. 1. The corresponding relationship between the focal plane of the HR camera's CCD and output data

This paper proposes an automated seamless mosaicking method to stitch the 6 strips of images according to the characteristics of data from CBERS-02C. As mentioned previously, the satellite is also equipped with a PAN camera. The PAN camera contains only a single CCD array, and has a very close imaging time to HR camera, and the coverage of the camera is slightly wider than HR images. Therefore, it is practical to make the $6 \mathrm{HR}$ strip images geometrically conforming to the PAN image obtained from the same area, then the problem of mosaicking could be settled in the course of nature. Owing to the image distortions arising from variety of factors, geometrically correction is adopted before image mosaicking in many applications [3]-[5]. Taking this problem into consideration, our approach also aims to generate a seamless stitching HR image after geometric correction. 
The developed algorithm automatically stitches the $6 \mathrm{HR}$ strips of images under the premise of the precise orientation to the PAN image. The entire process is shown in Fig.2. The data including 6 strips of images with corresponding RPC (Rational Polynomial Coefficients) parameters need to be prepared, and the output result is a single scene of HR image after geometric correction.

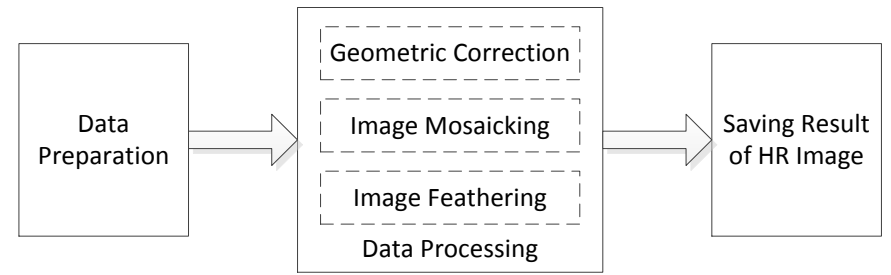

Fig. 2. Process chain for the generation of mosaicked HR image

\section{Methodology}

An approach is proposed that automatically corrects multi strips of images and seamlessly stitches the images without any second resampling of the data. The entire process employs the following technical methods: (1) Image geometric correction based on RFM, (2) image mosaicking and (3) weight-based image feathering.

\section{A. Image Geometric Correction Based on RFM}

The primary step during image geometric correction, is to establish a mapping relationship between the object-space coordinates and image-space coordinates. The Rational Function Model could be used to represent this relationship.

The RFM uses the ratio of two cubic polynomials to represent the rigorous geometric sensor model of satellite imagery. The model has the following general form as presented by Grodecki and Dial [6]:

$$
\left\{\begin{array}{l}
S_{r}=\frac{\operatorname{Num}_{L}(P, L, H)}{\operatorname{Den}_{L}(P, L, H)} \\
S_{c}=\frac{\operatorname{Num}_{S}(P, L, H)}{\operatorname{Den}_{S}(P, L, H)}
\end{array}\right.
$$

Where $\left(S_{r}, S_{c}\right)$ and $(P, L, H)$ are the normalized coordinates of the image-space and object-space points, respectively.

The second step is gray resampling to fill in the empty matrix created in the object-space. The gray value of every output matrix pixel is determined by the values of the pixels around the corresponding position in the image-space. The kernel adopted for resampling in our approach is bilinear interpolation [7].

Since the RPC parameters of strip images used for correction has been refined by the precise orientation to the PAN image, the strip images after correction are supposed to have consistent accuracy with PAN image.

\section{B. Image Mosaicking}

Except for A3 and B1, the rest of corrected strip images both have a very limited range of overlapping area (Fig.3). The implementation of conventional mosaic algorithm will be quite complicated in such a case. In this paper, an approach that combines geometric correction and seamless stitching as one step is presented to generate a mosaicked HR image efficiently.

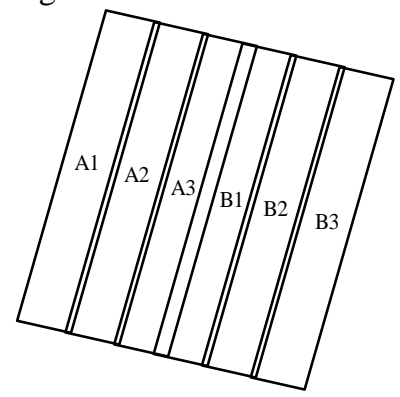

Fig. 3. The positional relationship of 6 corrected strip images

Image geometric correction is actually a process of establishing a spatial correspondence between image-space coordinates and object-space coordinates of the image. For a single image, one ground point has only one corresponding point in the image-space at most. However, if there are multiple images having a certain overlap, there may be a situation that a ground point has more than one corresponding image points. Therefore, if only the correspondence relationship between a ground point and the image points (may be one point, may be more than one point existing on multiple images) is clarified, the gray value of the ground point can be determined from the most suitable image point, and then the mosaicked HR image can be obtained. As shown in Fig.3, the overlapping areas between adjacent images are both very limited. Two image points corresponding to a ground point will be the most extreme case. Once a ground point $\mathrm{P}$ has two corresponding image points $p_{1}$ and $p_{2}$ on strip images A1 and $\mathrm{A} 2$ respectively, the comparison of vertical distance to the edge of strip image will give the best choice from the candidate image points (Fig.4), which means, the gray value of image point with minimum distance will be chosen as value for the ground point.
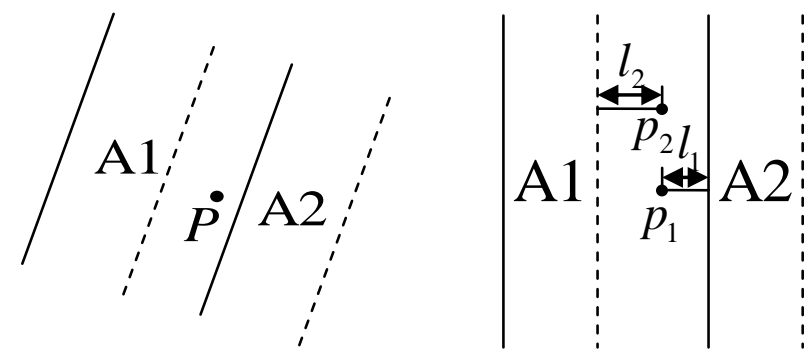

(a) the ground point on corrected image (b) image points on original images

Fig. 4. The corresponding relationship between the ground point and image point in overlapping area

In general, if there are over one image points corresponding to a ground point, the gray value of the ground point can be decided as follows:

$$
G(X, Y)= \begin{cases}g\left(x_{i}, y_{i}\right), & l_{i} \geq l_{j} \\ g\left(x_{j}, y_{j}\right), & l_{i}<l_{j}\end{cases}
$$


Where $G(X, Y)$ is the gray value of the ground point, $g\left(x_{i}, y_{i}\right)$ is the gray value for image point $p_{i}$, and $l_{i}$ is the vertical distance from point $p_{i}$ to the edge of strip image.

\section{Image feathering}

The mosaicked HR image calculated by Equation 1 and Equation 2 could not yet be submitted as a perfect product. There will be certain gray level difference in the connecting area of the edges of strip images, and an obvious jagged stitching line can be observed between image edges. The operation of image feathering should be adopted to solve this problem. For a HR image which has been already stitched, the implement of image feathering will be a tedious process, but if we choose to perform image feathering while image mosaicking is in progress, the problem will be easily solved by only improving Equation 2 with weighted distance method [8]. The improved equation is as follows:

$$
G(X, Y)= \begin{cases}g\left(x_{i}, y_{i}\right), & d>w \\ g\left(x_{j}, y_{j}\right), & d<-w \\ \frac{(w+d) g\left(x_{i}, y_{i}\right)+(w-d) g\left(x_{j}, y_{j}\right)}{2 w}, & |d| \leq w\end{cases}
$$

Where $d=\left(l_{i}-l_{j}\right) / 2$, which can be interpreted as the vertical distance from image point to the stitching line, and $2 w$ is the chosen transition width of feathering region.

\section{One-Step Processing for Generation of HR Image}

Combined with Equation 1 and Equation 3, the process of geometric correction, image mosaicking and image feathering can be completed in one step without any second resampling. In general, the entire process could be represented as the following steps:

1) Scope Determination: Calculate the geographical scope of every strip image with the RPC parameters and the value of ground sampling distance. Take the union scope as the scope of HR image.

2) Valid Strip Image Selection: For every pixel of HR image, calculate the image-space coordinates on each strip image with its object-space coordinates and RPC parameters. If the position of the image-space coordinates is within the scope of the strip image, the image is then treated as a valid image.

3) Gray Value Assignment: If the number of valid image is equal to 0 or greater than 2 , it means that there is no valid images or there may be wrong coordinates calculated among the valid images, the process should be switched to step 2); If there is only one valid strip image, the gray value of the ground point can be directly assigned by the value of the image point, which is interpolated by the value of the pixels surrounding with it; If the number of valid image is 2 , the value of the two image points should be interpolated in advance, and then the ground point's gray value can be calculated by Equation 3 .
Repeat Step 2) to Step 3), and then the HR image can be generated till the image resampling is completely finished.

The geometric correction is executed by unit of image grid, to complete correction of a grid of image, only coordinates of four corner points should be calculated by RPC parameters, the rest of the points' coordinates can be obtained by interpolation. In addition, considering that there is no complicated calculation during the process of image mosaicking and image feathering, the entire procedure of generation of HR image can be fulfilled very efficiently.

\section{EXPERIMENTAL RESULTS}

Experiments were conducted by applying the proposed method to the multi-strip images from realistic data of CBERS02C satellite. The study area is located in Qinghai Province of China. Before using in the mosaicking test, the strip images have both been radiometrically corrected, and performed accurate orientation to the PAN image obtained from the same area. Figure 5 shows the data before processing and the mosaicked image after using the above mentioned approach. The mosaicked image is of size, $26992 \times 30951$ pixels and the ground sampling distance is $2.5 \mathrm{~m}$. On a general personal computer, the consuming time of the processing is less than 5 minutes. The efficiency is proven to be very high.

Figure 6 shows the stitching results in part of HR image (with or without image feathering process), it can be seen that the images were perfectly mosaicked, and the mosaicked region of images could have a smooth transition.

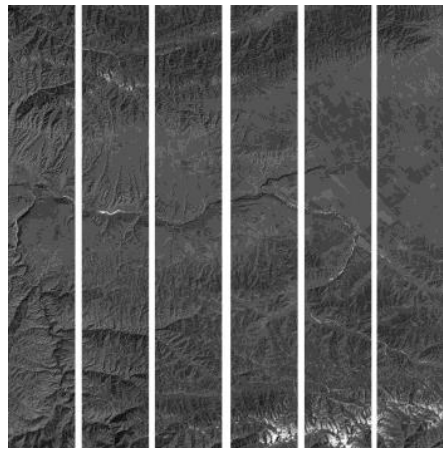

(a) Images after radiometric correction

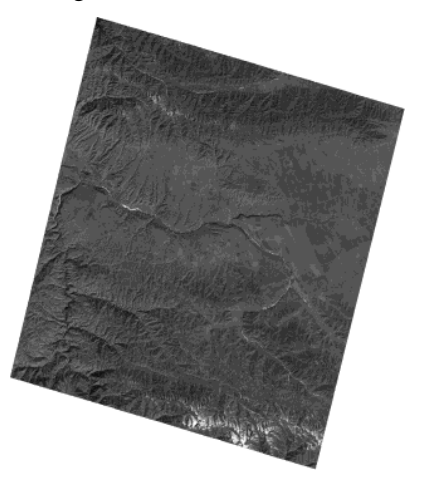

(b) Geometrically corrected, mosaicked images

Fig. 5. Separated strip images and mosaicked HR image 

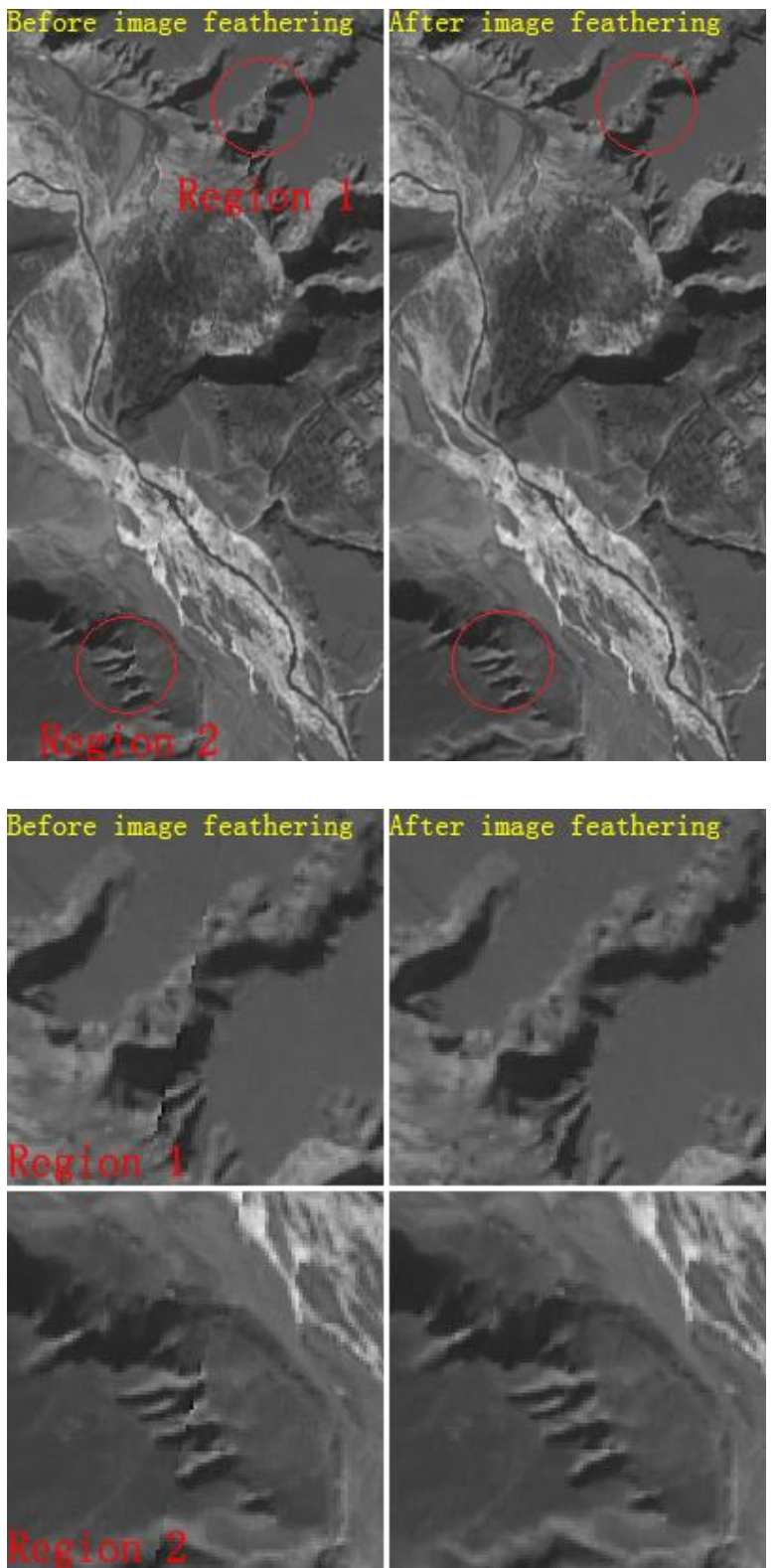

Fig. 6. Stitching results in part of HR image

\section{CONCLUSIONS}

In this paper an automated seamless mosaicking method for multi-strip data has been presented. The method is proven to be applicable to the generation of high-resolution geometrically corrected image from CBERS-02C imagery. Currently the presented method has also been successfully applied to the automatic data processing system of CBERS-02C satellite.

\section{ACKNOWLEDGMENT}

This work was supported by National Basic Research Program with project number 2012CB719904 and National HiTech Research and Development Program with project number 2013AA12A401.

\section{REFERENCES}

[1] S. Li, T. Liu and H. Wang, "Image Mosaic for TDICCD Pushbroom Camera Image Based on Image Matching," Remote Sensing Technology and Application, vol. 24(3), pp. 374-378, 2009(In Chinese).

[2] S. D. Naidu, B. G. Krishna, A. Gupta, T. P. Srinivasan and P. K. Srivastava, "Strip Mosaicing of Satellite Data Having Step and Stare Imaging," Indian Cartographer, vol. 12, pp. 67-72, 2002.

[3] R. Ramakrishnan, S. M. Moorthi, N. Padmanabhan and P. Gupta "An automated seamless mosaicing system of multi-charge coupled devices of panchromatic data," Journal of the Indian Society of Remote Sensing, vol. 32(1), pp. 103-111, 2004.

[4] B. Wu, J. Yang, J. Chen, J. Chen and J. Wu, "A Method of Obtaining Accurate Active Area of Remote Sensing Image and Application in Mosaicking," Remote Sensing, Environment and Transportation Engineering (RSETE), 2012 2nd International Conference on. IEEE, pp. 1-4, June 2012.

[5] L. Han, N. Guo, Y. Li, P. Ma and D. Yin, "The dynamically remote sensing monitoring and impacts of climatic change on Maqu wetland in 2002-2010," In Geoscience and Remote Sensing Symposium (IGARSS), 2012 IEEE International. IEEE, pp. 6115-6117, July 2012.

[6] J. Grodecki and G. Dial, "Block adjustment of high-resolution satellite images described by rational functions," Photogramm. Eng. Remote Sens., vol. 69, no. 1, pp. 59-68, Jan. 2003.

[7] R. C. Gonzalez and R. E. Woods, Digital Image Processing, 2nd Edition, Prentice-Hall, New Jersey, 2002.

[8] W. Huang, S. Zhu and H. Chen, "Seamline Removing in Mosaicked Image," Journal of Institute of Surveying and Mapping, vol. 17, no. 1, pp. 31-33, 2000. 